\title{
El ceftriaxone IM monodosis fue tan eficaz como la trimetroprima-sulfametoxazol oral por 10 días en el tratamiento de la otitis media aguda en niños
}

E.Barnett, D.Teele, J.Klein et al. Comparison of Ceftriaxone and Trimetoprim-Sulfametoxazol for Acute Otitis Media. Pediatrics 1997 Vol 99: 23-28

\section{Objetivo}

Comparar en el tratamiento de la otitis media aguda (OMA) en niños la eficacia entre una sola dosis (IM) de ceftriaxone (CFX) $(50 \mathrm{mg} / \mathrm{kg})$ y diez días de tratamiento oral en dos dosis con cotrimoxazol ( $8 \mathrm{mg} / \mathrm{kg} /$ dia de TMP y $40 \mathrm{mg} / \mathrm{kg} /$ dia de SMZ.).

\section{Diseño}

Ensayo clínico, randomizado, simple ciego con poder del $80 \%$ para detectar una diferencia del $10 \%$ entre ambos grupos.

\section{Lugar}

Boston, E.E.U.U

\section{Pacientes}

596 niños entre 3 meses y 3 años de edad reclutados en centros de atención primaria.

Criterios de exclusión: Antibióticos en los 7 dias previos, anomalías anatómicas, inmunosupresión, enfermedad crónica, alergia a penicilinas, cefalosporinas o sulfas, antecedente de timpanostomia. OMA se definió como signos de enfermedad aguda, membrana timpánica anormal y signos objetivos de derrame en oido medio.

\section{Intervención}

484 pacientes ( $82 \%$ ) fueron considerados elegibles. 241 recibieron CFX y 243 TMP-SMZ. Los grupos estuvieron bien balanceados. Fueron recitados al dia 3, 14 y 28. Al día 3 se los consideró curados si los signos de enfermedad habían desaparecido y se lo veía en su estado habitual de salud; mejorado si tenía menos signos, si estaba afebril y si el padre o el pediatra lo veían mejor. Se consideró que había fallado el tratamiento si persistían los signos o había nuevos. A los días 14-28 se los consideró curados si permanecían bien desde la visita previa. Se consideró que había fallado el tratamiento, si había nuevos signos más la persistencia de derrame.

\section{Resultados}

El análisis fue hecho por intención de tratar al grupo inicial de 484 niños seguido del día 0 al 14 inclusive. Los resultados fueron equivalentes $(\mathrm{P}=0.29)$. No hubo diferencias significativas en cuanto a la persistencia de derrame en el oído medio a.los 14 y 28 días. Efectos adversos: el $23.6 \%$ del grupo CFX y el $9.2 \%$ del grupo cotrimoxazol presentó diarrea $(\mathrm{P}<0.01)$. El $8.4 \%$ del grupo CFX presentó dolor en el sitio de inyección.

\section{Conclusiones}

La eficacia del CFX monodosis IM es comparable a la de 10 días de TMP-SMZ oral.

Apoyo económico: Laboratorio Roche.

\section{Comentario}

Los tratamientos de diez días de duración son los habituales para el tratamiento de la otitis media, sin embargo, con el objetivo de bajar los costos y aumentar la adherencia se han ensayado otras propuestas terapéuticas. En este trabajo se usó TMPSMZ como droga de comparación por $\mathrm{su}$ eficacia frente a gérmenes productores de B lactamasas y porque era el preferido de los pediatras participantes. Teniendo en cuenta la eficacia de ambas estrategias al día 14, analizadas por intención de tratar, podemos suponer que al margen de la adherencia que haya habido en el grupo de diez días de tratamiento, el resultado fue equivalente, o sea que la estrategia de indicar un tratamiento o el otro tendría el mismo resultado. Una limitación a considerar en este punto es que el poder (capacidad que tiene un estudio para detectar diferencias verdaderas entre los grupos) fue del $80 \%$, y en un estudio de equivalencia en el cual la hipótesis es que no hay diferencia en los tratamientos, el poder debería ser mayoral 90\%. La consecuencia de esto es que el estudio no tenga la sensibilidad suficiente para detectar diferencias reales entre los grupos.
También en general el cumplimiento del tratamiento en un trabajo de investigación suele ser mayor que en la práctica clínica. En cuanto al costo del tratamiento en nuestro medio, es importante destacar que para niños menores de $10 \mathrm{~kg}$. 1os costos son equivalentes: Ceftriaxone $\$ 15$ y TMP SMZ $\$ 14$; siendo más caro el tratamiento con Ceftriaxone en los de mayor peso:Ceftriaxone \$26 y TMP SMZ \$14.

Dr. Sergio Terrasa Unidad de Medicina Familiar y Preventiva Hospital Italiano de Buenos Aires 